\title{
A symmetry for vanishing cosmological constant in an extra-dimensional toy model
}

\author{
Recai Erdem \\ Department of Physics, İzmir Institute of Technology, Gülbahçe Köyü, Urla, İzmir 35430, Turkey \\ Received 11 March 2005; received in revised form 9 June 2005; accepted 11 June 2005 \\ Available online 22 June 2005 \\ Editor: M. Cvetič
}

\begin{abstract}
We introduce a symmetry principle that forbids a bulk cosmological constant in six and ten dimensions. Then the symmetry is extended in six dimensions so that it insures absence of 4-dimensional cosmological constant induced by the six-dimensional curvature scalar, at least, for a class of metrics. A small cosmological constant may be induced in this scheme by breaking of the symmetry by a small amount.

(c) 2005 Elsevier B.V. All rights reserved.
\end{abstract}

Cosmological constant problem is a long standing problem [1]. The problem can be stated as the huge discrepancy between the observational and the theoretically expected values of the cosmological constant [2] and the lack of understanding of its extremely small value [3]. Numerous schemes, to solve this problem, range from the models which employ supersymmetry, supergravity, superstrings, anthropic principles, modified general relativity, self-tuning mechanisms, quantum cosmology, extra dimensions, and combinations of these ideas [2,4-7]. Although they shed some light on the direction of the solution of this problem, they have not given a wholly satisfactory, widely accepted answer to this question. Among these attempts extra-dimensional models become more popular because they give model builders more flexibility [5-7]. This is mainly due to the fact the no-go theorem of Weinberg [2] is intrinsically four-dimensional; for example, the equations of motion for a field constant in 4 dimensions may contain a contribution from extra-dimensional kinetic term in the Lagrangian hence making the Weinberg's argument non-applicable in higher dimensions. Moreover, the models where a four-dimensional space is embedded in a higher-dimensional space may have striking differences. For example, four-dimensional world may be embedded in extra dimensions in such a way that the 4-dimensional brane remains flat under energy density changes on the brane through the counter balance of the curvature due to the extra dimensions and the brane tension [7]. However, these models, although appealing, at present have some technical problems such as need for large extra

E-mail address: recaierdem@iyte.edu.tr (R. Erdem). 
dimensions which may be in conflict with astronomical data, fine tuning, technicalities with quantum loop corrections, severe restrictions on dilaton-brane couplings [8]. So, additional insight on the cosmological constant in extra dimensions may be useful. In this Letter we study the implications of a symmetry, similar to scale invariance with a complex scale factor, on the cosmological constant. In fact, it seems that such a symmetry principle was also noticed by 't Hooft (though unpublished) [9]. We find that this symmetry forbids a non-zero bulk cosmological constant in 6 and 10 dimensions. We consider the 6-dimensional case in this Letter. We extend the symmetry so that the contribution to the cosmological constant due to the extra-dimensional curvature scalar vanishes as well. We find that breaking of the symmetry by a small amount may result in a small cosmological constant in this scheme. We also briefly discuss the restriction put on the form of matter Lagrangian by this symmetry.

Consider the transformation which multiplies the coordinates by the imaginary number $i$

$$
x_{A} \rightarrow i x_{A}, \quad A=0,1, \ldots, D-1,
$$

where $D$ stands for the dimension of the space. This transformation may be viewed as an analytic continuation followed by a rotation by $\pi / 2$ in each complex plane. We impose the symmetry

$$
g_{A B} \rightarrow g_{A B} \quad \text { as } x_{A} \rightarrow i x_{A}, A=0,1, \ldots, D-1 .
$$

Under Eq. (2) the scalar curvature is multiplied by -1

$$
R \rightarrow-R,
$$

and

$$
\begin{aligned}
d^{D} x & \rightarrow d^{D} x \quad \text { if } D=4 n \\
& \rightarrow-d^{D} x \quad \text { if } D=2(2 n+1) \\
& \rightarrow \pm i d^{D} x \quad \text { if } D=2 n+1 \\
n & =0,1,2,3, \ldots
\end{aligned}
$$

The requirement that Einstein-Hilbert action

$$
S_{R}=\frac{1}{16 \pi G} \int \sqrt{g} R d^{D} x
$$

should be invariant under (2) selects out

$$
D=2(2 n+1), \quad n=0,1,2,3, \ldots
$$

In fact, in the case of exact symmetry the action should be invariant up to a multiplicative constant because the equations of motion remain the same. However, if the symmetry is broken (even by a small amount which is the physical situation) then the part of the action respecting the symmetry must be strictly invariant since each constant multiplying the symmetry preserving part of the action leads to a different equation of motion in general after taking the symmetry breaking part into account. Another point worthwhile to mention is that under Eq. (2)

$$
d s^{2} \rightarrow-d s^{2}
$$

This implies a symmetry under exchange of space-like and time-like intervals. The implications of this transformation need a separate study.

We notice from Eqs. (3) and (5) that the cosmological constant term for the action

$$
S_{C}=\frac{1}{16 \pi G} \int \sqrt{g} \Lambda d^{D} x
$$


(where $\Lambda$ is constant in $x_{A}, A=0,1, \ldots, D-1$ ) is not allowed by the symmetry induced by Eq. (1) for $D$ 's satisfying (8). Under the requirement $D \geqslant 4$ and $D \leqslant 10$ (which comes from string theory) the only possible dimensions allowed by the symmetry induced by (2) are 6 and 10 .

In this Letter we study the minimal case, i.e., $D=6$ case. It is evident that an $S_{R}$ term is not allowed in $D=4$. On the other hand, a cosmological constant term, Eq. (10), is allowed in 4 dimensions. In other words, although the invariance of the action under (2) forbids a six-dimensional cosmological constant it does not forbid a 4dimensional one. So a 4-dimensional cosmological constant may be induced through the six-dimensional curvature scalar even if there is no contribution to it through a six-dimensional bulk cosmological constant. A $D$-dimensional curvature scalar $(D>4)$ may be written as

$$
R=R_{1}\left(x_{\mu}, x_{a}\right)+R_{2}\left(x_{a}\right), \quad \mu=0,1,2,3, \quad a=4,5, \ldots, D-1,
$$

where $R_{2}$ is the part of the curvature scalar which is independent of $x_{\mu}$ (i.e., $R_{2}$ depends only on the extra dimensions) and $R_{1}$ is the part which contains $x_{\mu}$-dependent and the mixed terms. A non-vanishing $R_{2}$ in general introduces a cosmological constant in 4 dimensions. So one must impose a symmetry which eliminates $R_{2}$ as well in order to make the 4-dimensional cosmological constant zero altogether.

The local 4-dimensional Poincaré invariance in six dimensions results in the metric [10]

$$
d s^{2}=\sigma\left(x^{a}\right) g_{\mu \nu} d x^{\mu} d x^{\nu}+g_{a b}\left(x^{a}\right) d x^{a} d x^{b}, \quad \mu \nu=0,1,2,3, \quad a, b=4,5 .
$$

In addition to the symmetry (2) we require

$$
g_{A B} \rightarrow g_{A B} \quad \text { as } x_{4} \leftrightarrow x_{5}
$$

and take

$$
g_{44}=-g_{55}
$$

(which may be obtained by imposing $g_{a b} d x^{a} d x^{b} \rightarrow g_{a b} d x^{a} d x^{b}$ under Eq. (2)). Under these requirements we find that the extra-dimensional components of the Riemann tensor are zero, $R_{a b}=0$, and its 4-dimensional part $R_{\mu \nu}$ depends only on the 4-dimensional coordinates $x^{\mu}$. So $R_{2}\left(x^{a}\right)=0$ in this case. In other words the local 4-dimensional Poincare invariance together with the requirements Eqs. (13) and (14) guarantee the absence of a contribution from 6-dimensional curvature scalar to the 4-dimensional cosmological constant.

We have introduced the symmetry (2) to eliminate the 6-dimensional bulk cosmological constant and the symmetry (13) and (14)) to eliminate a possible contribution to the 4-dimensional cosmological constant from 6-dimensional curvature scalar. Now we give some examples first to see the picture more clearly and then consider the case of the symmetry breaking by a small amount. First take the metric

$$
\begin{gathered}
d s^{2}=\Omega_{1}^{2}(y) g_{\mu v}(x) d x^{\mu} d x^{v}+\Omega_{2}^{2}(y) \eta_{a b} d y^{a} d y^{b}, \quad\left(\eta_{a b}\right)=\operatorname{diag}(-1,1), \\
\mu, v=0,1,2,3, \quad a, b=1,2, \quad y_{1}=x_{4}, \quad y_{2}=x_{5},
\end{gathered}
$$

where $x$ stands for the 4-dimensional coordinates and $y$ for the extra-dimensional coordinates. Provided that the metric tensor is a smooth function of $y$ the curvature scalar corresponding to (15) is

$$
\begin{aligned}
R= & \Omega_{1}^{-2} g^{\mu \nu} R_{\mu \nu}-\Omega_{1}^{-2}\left[10 \eta^{a b} \partial_{a} \partial_{b}\left(\ln \Omega_{1}\right)+20 \eta^{a b} \partial_{a}\left(\ln \Omega_{1}\right) \partial_{b} \Omega_{1}\right] \\
& +2 \Omega_{2}^{-2}\left[\eta^{a b} \partial_{a} \partial_{b}\left(\ln \Omega_{1}\right)-\eta^{a b} \partial_{a} \partial_{b}\left(\ln \Omega_{2}\right)\right] .
\end{aligned}
$$

Let us consider the case where

$$
\Omega_{1}=\Omega_{1}\left(k_{1} y_{1}+k_{2} y_{2}\right), \quad \Omega_{2}=\Omega_{2}\left(k_{3} y_{1}+k_{4} y_{2}\right), \quad y_{1}=x_{4}, \quad y_{2}=x_{5} .
$$


Then the curvature scalar is obtained as

$$
\begin{aligned}
& R= \Omega_{1}^{-2} g^{\mu \nu} R_{\mu \nu}-\Omega_{1}^{-2}\left(k_{1}^{2}-k_{2}^{2}\right)\left[10 \frac{d^{2}\left(\ln \Omega_{1}\right)}{d u_{1}^{2}}+20\left(\frac{d \ln \Omega_{1}}{d u_{1}}\right)^{2}\right] \\
&+2 \Omega_{2}^{-2}\left(k_{1}^{2}-k_{2}^{2}\right) \frac{d^{2}\left(\ln \Omega_{2}\right)}{d u_{1}^{2}}-2 \Omega_{2}^{-2}\left(k_{3}^{2}-k_{4}^{2}\right) \frac{d^{2}\left(\ln \Omega_{2}\right)}{d u_{2}^{2}}, \\
& u_{1}=k_{1} y_{1}+k_{2} y_{2}, \quad u_{2}=k_{3} y_{1}+k_{4} y_{2} .
\end{aligned}
$$

Because $k_{1}, k_{2}$ are projected out by $\partial_{1}, \partial_{2}$ they transform under (1) like $\partial_{1}, \partial_{2}$; respectively,

$$
k_{a} \rightarrow-i k_{a} \quad \text { as } x_{a} \rightarrow i x_{a}, a=1,2 .
$$

So, $k_{1} y_{1}+k_{2} y_{2}$ is automatically invariant under (1) hence $\Omega_{1}, \Omega_{2}$ automatically obey (2). The application of the requirement, Eq. (13) to $\Omega_{1}$ and $\Omega_{2}$ results in

$$
k_{1}=k_{2}, \quad k_{3}=k_{4} .
$$

So the extra-dimensional contribution to the curvature scalar, i.e., $R_{2}$ in (11) vanishes. In other words, the symmetry (13) and (14) requires the contribution to the cosmological constant from the extra-dimensional curvature scalar be zero as well. The metric given in (16) is a smooth function of $x_{4}, x_{5}$. So the form of metric and the fact one of the extra dimensions is space-like and the other is space-like [11] brings the over-all factors of $k_{1(3)}^{2}-k_{2(4)}^{2}$, which vanish in the limit of the symmetry (14) to make the curvature scalar zero. However, if the metric tensor is not a smooth function of $x_{A}$ then $R_{2}$ does not have $k_{1(3)}^{2}-k_{2(4)}^{2}$ as over-all factors, however, $R_{2}$ is still zero. To be more specific we consider a metric of the form of (15) and (16) with

$$
\Omega_{1}^{2}=\operatorname{Cos}\left(\left|k_{1} y_{1}\right|+\left|k_{2} y_{2}\right|\right), \quad \Omega_{2}^{2}=0, \quad y_{1}=x_{4}, \quad y_{2}=x_{5},
$$

where a $Z_{2} \times Z_{2}$ orbifold symmetry induced by $k_{1} y_{1} \rightarrow-k_{1} y_{1}, k_{2} y_{2} \rightarrow-k_{2} y_{2}$, to get the absolute value signs in (20) and two branes located at the points $k_{1} y_{1}=0, k_{2} y_{2}=0, k_{1} y_{1}=\pi, k_{2} y_{2}=\pi$. By using Eq. (17) we obtain the curvature scalar as

$$
R=\frac{1}{\operatorname{Cos}\left(\left|k_{1} y_{1}\right|+\left|k_{2} y_{2}\right|\right)}\left[g^{\mu v} R_{\mu \nu}+10 k_{1}^{2} \tan u \tilde{\delta}_{1}-10 k_{2}^{2} \tan u \tilde{\delta}_{2}+5\left(k_{1}^{2}-k_{2}^{2}\right)\right], \quad u=\left|k_{1} y_{1}\right|+\left|k_{2} y_{2}\right|,
$$

where $\tilde{\delta}_{1}=\delta\left(k_{1} y_{1}\right)-\delta\left(k_{1} y_{1}-\pi\right), \tilde{\delta}_{2}=\delta\left(k_{2} y_{2}\right)-\delta\left(k_{2} y_{2}-\pi\right)$, and $g^{\mu v} R_{\mu \nu}$ depends only on $x_{\mu}$. Each delta function defines a 5-dimensional subspace and the intersections of these 5-dimensional subspaces define four 3branes which consist of two pairs of 3-branes related by the reversal of their signatures. We see that $R_{2}$ due to Eq. (21) is zero in this case as well when $k_{1}=k_{2}$ (i.e., when the symmetry in Eq. (14) is exact) after $R$ is integrated over $y_{1}$ and $y_{2}$.

One must break this symmetry by a small amount in order to get a small cosmological constant in agreement with observations. First we consider the metric (20). Assume that the symmetry imposed by (14) is broken for the metric (20) by a small amount $k_{1}-k_{2}=\Delta$. Then the 4-dimensional cosmological constant induced by $R_{2}$ is approximately equal to

$$
\frac{2 k_{1} \Delta}{16 \pi G} \int 5 \operatorname{Cos}^{2} u d y_{1} d y_{2}=\frac{5 \pi k_{1} \Delta}{4 G\left|k_{1}\right|^{2}} .
$$

We notice that the induced cosmological constant

$$
\Lambda^{(4)}=\frac{20 k_{1} \pi^{2} \Delta}{\left|k_{1}\right|^{2}}
$$


will be even closer to zero if $k_{1} \approx k_{2}$ is large and the smallness of the cosmological constant is protected by the symmetry. We have shown that the breaking of the symmetry for the metric (20) leads to a small cosmological constant provided the symmetry is broken by a small amount. In other words, a small breaking of the symmetry does not lead to a big cosmological constant. Now we get a more general conclusion for the more general class of metrics (12). The Einstein equations corresponding to a conformally transformed metric $\Omega^{2} g_{\mu \nu}$ relate to the Einstein equations corresponding to the original metric in six dimensions as

$$
\tilde{G}_{A B}=G_{A B}+4 \delta_{A}^{a} \delta_{B}^{b}\left(\partial_{a} \ln \Omega \partial_{b} \ln \Omega-\partial_{a} \partial_{b} \ln \Omega\right)+\tilde{g}_{A B}\left(6 \eta^{a b} \partial_{a} \ln \Omega \partial_{a} \ln \Omega+4 \eta^{a b} \partial_{a} \partial_{b} \ln \Omega\right),
$$

where $\tilde{G}_{A B}=\tilde{R}_{A B}-\frac{1}{2} \tilde{g}_{A B} \tilde{R}$ is the Einstein tensor corresponding to $\tilde{g}_{A B}=\Omega^{2} g_{A B}$ and $G_{A B}=R_{A B}-\frac{1}{2} g_{A B}$ is the Einstein tensor corresponding to $g_{A B}$. The terms containing $\Omega$ on the right-hand side of (24) may be identified as the terms corresponding to the energy-momentum tensor induced by the conformal transformation. Meanwhile, we observe that Dirac delta function can be written as

$$
\lim _{\alpha \rightarrow \infty} \alpha\left[1-\tanh ^{2}(\alpha z)\right]=\delta(z),
$$

which follows from the fact that the derivative of step function gives the Dirac delta function. If we let $\ln \Omega=$ $\beta \ln \operatorname{Cosh} \alpha\left(y_{1}-y_{0}\right)$ then the non-vanishing terms in Eq. (24) give

$$
\partial_{1} \ln \Omega \partial_{1} \ln \Omega \rightarrow \beta^{2} \alpha^{2} \quad \text { and } \quad \partial_{1} \partial_{1} \ln \Omega \rightarrow \beta \delta\left(y_{1}-y_{01}\right) \quad \text { as } \alpha \rightarrow \infty .
$$

A small $\beta$ corresponds to the breaking of the symmetry by a small amount. If we take $\beta=\epsilon \frac{1}{\alpha}$, where $\epsilon \ll 1$ then a small perturbation in energy-momentum distribution leads to an even smaller bulk cosmological constant and results in an over-all rescaling of the metric by $\Omega$.

The restriction put on the form of the matter Lagrangian by the symmetry Eq. (2) can be determined by requiring the invariance of the corresponding action which requires the Lagrangian $\mathcal{L}$ transform as $\mathcal{L} \rightarrow(-i)^{D} \mathcal{L}$. Then the transformation rule for the scalar field follows as

$$
\frac{1}{2} g^{A B} \partial_{A} \phi \partial_{B} \phi \rightarrow(-i)^{D} \frac{1}{2} g^{A B} \partial_{A} \phi \partial_{B} \phi \quad \text { implies } \phi \rightarrow(-i)^{\frac{D-2}{2}} \phi .
$$

The mass term, $\frac{1}{2} m^{2} \phi^{2}$ is compatible with this symmetry since $m^{2} \rightarrow-m^{2}$ (which follows from $p^{2}=m^{2}$ ). However, a $\phi^{4}$ term is not compatible with this symmetry (unless $D=4$ ). So this symmetry implies that $\phi^{4}$ terms may be induced only on 4-dimensional branes. This together with the zero (or almost zero) value of cosmological constant requires a two branes (or even number of branes) scenario, where $\phi^{4}$ terms are induced at both of the branes simultaneously and their contribution cancel (or almost cancel) after integrated out over the extra dimensions. The transformation rule for fermions follows as

$$
i \bar{\psi} \gamma^{A} \partial_{A} \psi \rightarrow(-i)^{D} i \bar{\psi} \gamma^{A} \partial_{A} \psi \quad \text { implies } \psi \rightarrow(-i)^{\frac{D-1}{2}} \psi .
$$

The mass term $m \bar{\psi} \psi$, the fermion-scalar interaction term and the fermion-gauge boson interaction term $i \bar{\psi} \gamma^{A} B_{A} \psi$, all are compatible with the symmetry, while

$$
\bar{\psi} \psi \phi \rightarrow(-i)^{\frac{3 D-4}{2}}
$$

is compatible with the symmetry only for $D=4$. So this term may only be induced on a 4-dimensional brane. Because $\partial_{A}$ and $B_{A}$ are combined in the covariant derivative $\partial_{A}-i B_{A}, B_{A}$ must transform in the same way as $\partial_{A}$. This implies that the gauge field kinetic term $-\frac{1}{4} F_{A B} F^{A B}$

$$
F_{A C} F^{A C}=\left(\partial_{A} B_{C}-\partial_{C} B_{A}\right)\left(\partial^{A} B^{C}-\partial^{C} B^{A}\right) \rightarrow(-i)^{D} F_{A C} F^{A C}
$$

is also compatible with the symmetry.

A comment is in order at this point. We have found in the above paragraph that the mass terms are allowed in all dimensions unlike the usual scale invariant field theories and the result of Nobbenhuis [9] although he uses 
the same symmetry as the one given here. The difference between the conclusions come from the difference in the identification of how mass terms behave under the scaling transformation. In the usual scale invariance and in the Nobbenhuis's study [9] masses are taken to be invariant under the symmetry transformation while in this study the masses transform like momenta $\left(p^{2}=m^{2}\right)$. In fact, the approach in the present study is in agreement with the identification of mass terms as the kinetic terms of the higher dimensions and under this condition this is the only consistent transformation provided that one scales all the dimensions simultaneously. Otherwise, it means that either one does not scale all the dimensions or does not consider the mass terms as the kinetic terms of higher dimensions. In fact, the difference in the approach to the scaling property of mass term is just a matter of convenience. It depends on one's aim of using the scale invariance. If one just tries to get phenomenological results confined to relatively low energies where the extra dimensions related to the masses are not observable one should take the masses be invariant under scale transformation. However, if one tries to get general results applicable to all dimensions (as is the case in this study) one should transform the mass terms like momenta because it is just the kinetic term written in another form in this case. Another difference between the result of Nobbenhuis and mine is that he reaches the conclusion that the cosmological constant must vanish in the usual four dimensions, $D=4$, while I obtain the same result for $D=2(2 n+1)$, i.e., $D=2,6,10$. This difference is due to different methods employed in the implementation of the symmetry. Nobbenhuis uses the covariance of the equations of motion (i.e., Einstein field equations) under the symmetry employed in this Letter while I use the invariance of the action functional under the same symmetry as the basis of my arguments. The requirement, of the covariance of the Einstein equations, used in Nobbenhuis's study leads to the result that the Einstein tensor and cosmological constant cannot coexist, either of them must vanish. This method does not tell anything about the allowed number of dimensions. The conclusion of Nobbenhuis depends on the assumption that the Einstein tensor is already allowed in four dimensions, so the cosmological constant must vanish. On the other hand, the requirement of the invariance of the action, used in the present study, does not only give the result that both of the cosmological constant and the Einstein tensor cannot coexist but it also leads to the formula for the number of dimensions, $D=2(2 n+1)=2,6,10, \ldots$, which forbid a non-vanishing cosmological constant. In other words, in our analysis the Ricci scalar cannot be four-dimensional in origin, it must be induced, as an effective four-dimensional Ricci scalar, from higher dimensions (e.g., from a 6-dimensional Ricci scalar) so that it has some hidden invariance under the symmetry (through the extra-dimensional parameters which are integrated out).

Given the fact that the symmetry employed here is a sub-case of the complexified version of the scale invariance (hence, of the conformal invariance) and the fact that there are some serious problems with the quantization of the classical field theories with conformal invariance [12] (especially in dimensions other than two) one may wonder if a similar problem exists for this symmetry, that is, if the symmetry introduced in this Letter is preserved after quantization. It is a well-known fact that the lack of persistence of the scale invariance in quantum field theory results from the non-invariance of the correlation functions (i.e., $n$-point functions) under scale invariance. This non-invariance results in the nonconservation of the Noether current due to the breakdown of the scale symmetry after the quantization. This, in turn, induces conformal anomalies which are rather difficult to manage (especially in dimensions other than two). This situation is improved in the case of the symmetry introduced in this Letter. As it is evident from Eqs. (27), (28), (30) the 2-point functions (which serve as the building blocks for $n$-point functions) scale as $(-i)^{D-2}$ for scalars and gauge fields and scale as $(-i)^{D-1}$ for fermions. Therefore, the 2-point functions are invariant under this symmetry in the dimensions $D-2=4 n(D=4 n+2)$ for scalars and gauge fields and in $D-1=4 n(D=4 n+1)$ for fermions (where $n=0,1,2, \ldots)$. Hence one may speculate models where the renormalizability of the model for scalars-gauge fields and fermions is manifest at different dimensions higher than four (e.g., say, at $D=11$ or $D=7$ for fermions and at $D=10$ or $D=6$ for scalars and gauge fields) and at lower dimensions the theory behaves as an effective theory with a (in some sense) hidden symmetry. For the time being, these remarks are just speculations. A detailed study of this topic is necessary to arrive reliable conclusions about this point. In any case, with respect to quantization, this symmetry seems to be more promising than the usual scale symmetry. 
I hope that this study will give additional insight towards the solution of the cosmological constant problem. However, there is more work to be done in this direction. The metric employed here is static so the cosmological constant is constant in time. The need for inflation in the history of the universe needs a much larger value for the cosmological constant in the early universe. So, the next step should be making the metric time-dependent to get a time-dependent cosmological constant. The self-tuning solutions with large extra dimensions discussed in the introduction [8] need smaller sizes for the extra dimensions in the inflationary universe era to get larger cosmological constants in that era. On the other hand, the extra dimensions here may be small or large. Moreover, the extra dimensions in the inflationary era may be larger or smaller than their present values. For example, a term of $k_{0} t$ in the argument of the conformal term $\operatorname{Cos} u$ may break the symmetry thus induce a cosmological constant. The induced cosmological term would depend on the phase factor $k_{0} t$ hence it may be different at different times independent of the size of the extra dimensions. Another point, which needs further study, is the source of this symmetry and its breaking mechanisms both in a physical and a mathematical content. I hope the investigation of all these and other interesting unanticipated aspects of this symmetry give fruitful results.

\section{Acknowledgements}

I would like to thank Prof. Durmuş Ali Demir for reading the manuscript and fruitful discussions on the topic and for his valuable comments.

\section{References}

[1] N. Straumann, gr-qc/0208027.

[2] S. Weinberg, Rev. Mod. Phys. 61 (1989) 1.

[3] S. Eidelman, et al., Particle Data Group, Phys. Lett. B 592 (2004) 1.

[4] S. Mukohyama, L. Randall, Phys. Rev. Lett. 92 (2004) 211302.

[5] C.P. Burgess, R.C. Myers, F. Quevedo, Phys. Lett. B 495 (2000) 384.

[6] G. Dvali, G. Gabadadze, M. Shifman, Phys. Rev. D 67 (2003) 044020.

[7] N. Arkani-Hamed, S. Dimopoulos, N. Kaloper, R. Sundrum, Phys. Lett. B 480 (2000) 193; S. Kachru, M.B. Schulz, E. Silverstein, Phys. Rev. D 62 (2000) 045021;

A. Krause, JHEP 0309 (2003) 016;

Y. Aghababaie, C.P. Burgess, S.L. Parameswaran, F. Quevedo, Nucl. Phys. B 680 (2004) 389.

[8] S.M. Carroll, M.M. Guica, hep-th/0302067; C.P. Burgesss, Ann. Phys. 313 (2004) 283.

[9] After the submission of this work, hep-th/0410063, to the ArXive I have learnt from the paper given below that this symmetry principle was already noticed by G. 't Hooft (though unpublished): S. Nobbenhuis, gr-qc/0411064.

[10] V.A. Rubakov, M.E. Shaposhnikov, Phys. Lett. B 125 (1983) 139.

[11] The necessity of the presence of both time-like and space-like extra dimensions to make the cosmological constant zero for a static metric has been studied in: T. Li, Phys. Lett. B 503 (2001) 163.

[12] S. Coleman, Aspects of Symmetry, Cambridge Univ. Press, Cambridge, 1985; P. Di Francesco, P. Mathieu, D. Sénéchal, Conformal Field Theory, Springer-Verlag, New York, 1997. 